\title{
Study of strain-transfer of FBG sensors embedded in unidirectional composites
}

\author{
G. Pereira ${ }^{a, *}$, C. Frias ${ }^{a}$, H. Faria $^{a}$, O. Frazão ${ }^{b}$, A.T. Marques ${ }^{c}$ \\ a INEGI - Instituto de Engenharia Mecânica e Gestão Industrial, Campus FEUP, Rua Dr. Roberto Frias, 400, 4200-465 Porto, Portugal \\ ${ }^{\mathrm{b}}$ INESC Porto - Instituto de Engenharia de Sistemas e Computadores do Porto, Rua do Campo Alegre, 687, 4150-179 Porto, Portugal \\ ${ }^{\mathrm{c}}$ FEUP - Faculdade de Engenharia da Universidade do Porto, Rua Dr. Roberto Frias, s/n, 4200-465 Porto, Portugal
}

\section{A R T I C L E I N F O}

\section{Article history:}

Received 7 April 2013

Accepted 15 May 2013

\section{Keywords:}

Smart materials

Optical fibre Bragg grating sensors

Nondestructive testing

Structural health monitoring

\begin{abstract}
A B S T R A C T
Optical fibre Bragg grating (FBG) sensors are now quite established and widely used in strain measurements in composites. However, insufficient understanding of the limitations of the embedment and measuring techniques often leads to inaccurate results.

This work is a continuation of a novel method to improve the reliability and accuracy of the strain measurements on unidirectional composites using embedded FBG sensors [1]. A new combination of the pair host material/sensor was studied and characterized. Test specimens were manufactured with longitudinally embedded FBG sensors, using a glass/ epoxy prepreg system, in order to compare with a carbon/epoxy prepreg system. The combined behaviour of the sensors and the host material was characterized and a procedure to obtain a more accurate strain was defined for this new chosen material.
\end{abstract}

(c) 2013 Elsevier Ltd. All rights reserved.

\section{Introduction}

In recent years, the monitoring of composite materials and structures using optical fiber Bragg grating (FBG) sensors has increased substantially, as well as the more acceptance of FBG for quasi-distributed strain and temperature sensors [2,3]. The small diameter of the FBG sensors, the effective insulation, immunity to electromagnetic fields and the multiplexing ability, make them especially suitable for structural health monitoring. Therefore, this technology allows single or arrays of nearly non-intrusive sensors to be embedded in the composite material to be monitored.

The ability of these sensors to be part of the structure itself allows them to be used for online monitoring and inspections during the whole service life of the composite structure. Difficult handling and limited measuring range, fragile behaviour, coupled with mechanical and thermal sensibilities using conventional FBG sensors are some of

\footnotetext{
* Corresponding author. Tel.: +351 2295787 10; fax: +351 229537352 E-mail address: Gilmar.fepe@gmail.com (G. Pereira).
}

the disadvantages of this sensor technology. However, it is still very promising and several authors have been studying and further developing it [3-8].

The relationship between the wavelength shift and the real strain imposed on the sensor is accurately determined and catalogued for each grating. However, the accuracy of the measurement in real applications strongly depends on the effectiveness of the strain transfer from the host material to the FBG sensors. Typically, the optical fibre and the host material in which it is embedded have different material properties (such as longitudinal stiffness) and, therefore, strain in both materials will not be equal when load is applied [1,9].

Incomplete adhesion between the FBG sensor and the surrounding host material is a factor that reduces the quality of the measurement, also the surface size of the optical fiber may not be the most compatible with the matrix in which it will be embedded, so that deviations from the measured and the real strain field occur [10]. Nonlinearity in the sensor response can occur due to heterogeneity of the adhesion along the grating, thus adding further uncertainty to the measurement. 
In most of the applications of FBG to measure strains in composite materials, these issues are not taken into account and are neglected by most authors. Hence, the values retrieved from such measurements may be over or under estimating the real ones. Moreover, since normally the sensors are not calibrated for each material and/or application, the users are not aware of these errors in the measurements.

This study is a continuation of a previous work [1], focused on the application of FBG sensors in unidirectional (UD) composites to measure the longitudinal strain. The objectives were:

- To compare the mismatch between the real strain and that measured by the sensor, to different host materials (glass/epoxy and carbon/epoxy prepreg systems).

- To demonstrate that the difference between the stiffness between the FBG senor and the host material is the main cause of the non-perfect strain transfer.

- To determinate the correction factor and establish a procedure to obtain a more accurate strain value measured by the FBG sensor.

- To obtain a more accurate strain in a random application using a FBG sensor embedded in the composite material.

The experimental procedure to manufacture and test the specimens is the same as used in the previous study [1]. Also, the FBG sensors and the signal acquisition technology were the same as used in the previous study. The outputted wavelength signal was acquired at $1 \mathrm{~Hz}$ with a Braggmeter equipment, developed by FiberSensing ${ }^{\mathrm{TM}}$ (Fig. 1). A single mode (SM) FBG with one single grating, not-recoated, with grating length of $10 \mathrm{~mm}$ and fibre diameter of $125 \mu \mathrm{m}$, was used in each test specimen, and all gratings were nonrecoated in order to promote better strain transfer between the host material and the sensor.

\section{Material and experimental procedure}

In order to compare the strain transfer between the sensor and the host material, we used two materials with

\section{Table 1}

Properties of the Carbon fiber/epoxy prepreg and glass fiber/epoxy prepreg applied to the test specimens.

\begin{tabular}{lcc}
\hline & Glass fiber/epoxy & Carbon fiber/epoxy \\
\hline Filament diameter $[\mu \mathrm{m}]$ & 9 & 7 \\
Density $\left[\mathrm{g} / \mathrm{cm}^{3}\right.$ ] & $2.48-2.49$ & 1.79 \\
Tensile strength [MPa] & 4845 & 5000 \\
Tensile modulus [GPa] & $85.5-86.9$ & 245 \\
Number of filaments per tow & 6.000 & 24,000 \\
Nominal linear density [tex] & 363 & 1600 \\
\hline
\end{tabular}

different tensile modulus, glass fibre and carbon fibre. However, to have the same relationship in the sensor interface/host material we used the same matrix, epoxy prepreg system in both cases.

The characteristics of carbon fibre/epoxy prepreg and glass fibre/epoxy prepreg are presented in Table 1.

To produce the unidirectional composite specimens the same procedure as in the previous work was used [1]. The specimens were produced with a free length of $100 \mathrm{~mm}$, width of $20 \mathrm{~mm}$ and thickness of $1.5 \mathrm{~mm}$. Each test specimen consisted of two layers of six rovings of carbon fibre/ epoxy prepreg or glass fibre/epoxy prepreg each, and five valid specimens were manufactured. The samples were cured according to the temperature cycle specified by the manufacturer (90 min @ $150{ }^{\circ} \mathrm{C}$ ).

Similarly to the procedure to produce specimens, the experimental procedure used was the same as in the previous work [1]. A novel experimental procedure to test and accurately measure the longitudinal strain in UD composite laminates with embedded FBG sensors under tensile loading was drawn and validated, where the objective was to evaluate the mismatch between the strain measured by the FBG sensor and the real strain. To perform this analysis, the strain sensed by the FBG sensor was acquired using a Benchtop BraggMeter FS 5200 supplied by FiberSensing ${ }^{\mathrm{TM}}$ and synchronized with the strain measured by a universal strain gauge extensometer assembled in the universal testing machine Instron 4507. Then, the two values were compared in order to establish a calibration rule that

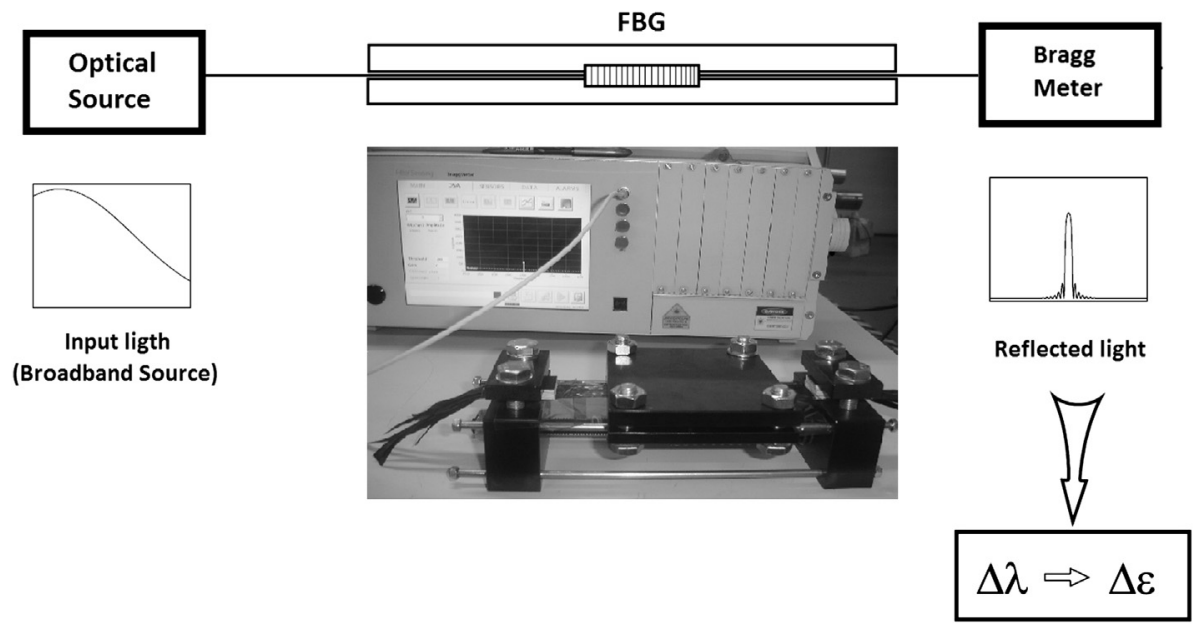

Fig. 1. Scheme of the data acquisition apparatus, using a Braggmeter. 


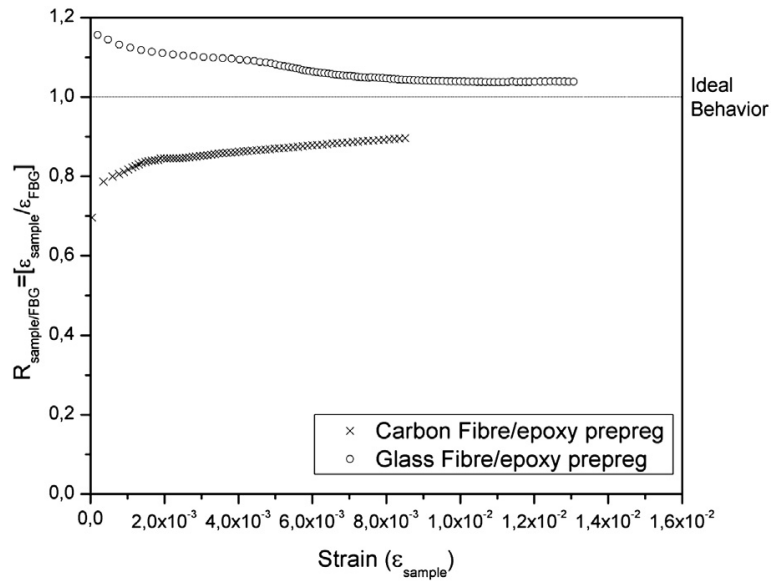

Fig. 2. Ratio between the real strain of the specimen and the strain measured by the FBG during the tensile test, for Glass/epoxy and Carbon/ epoxy systems.

matches with better accuracy the strain measured by the FBG sensor with the real behaviour of the host material.

\section{Discussion of results}

In Fig. 2, the relation between the strain measured by the FBG sensor and the real strain of the sample is shown,

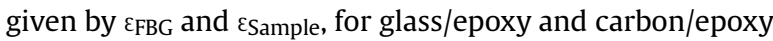
systems.

By assuming that the tests were performed at a constant temperature and, therefore, no temperature effects were introduced in the FBG sensing mechanism, the corresponding wavelength shift given by the following equation [4].

$\Delta \lambda_{B}=\lambda_{B}\left(\frac{1}{\Lambda_{B}} \frac{\partial \Lambda_{B}}{\partial \varepsilon}+\frac{1}{n_{0}} \frac{\partial n_{0}}{\partial \varepsilon}\right) \Delta \varepsilon=\lambda_{B}\left(1-p_{C}\right) \Delta \varepsilon$

where $\lambda_{B}$ is the wavelength, $\Lambda_{B}$ is the spacing between grating periods, $n_{0}$ is the effective index of the core and $p_{c}$ is the effective photoelastic coefficient of the optical fiber. For the FBG sensor used in this research, the wavelength-strain sensitivity at $\lambda_{B}=1550 \mathrm{~nm}$ is $1.1 \times 10^{-3} \mathrm{~nm} / \mu \varepsilon$ [11].

Analysing Fig. 2, we can verify that in the glass/epoxy prepreg system the strain given by the FBG sensor is lower than the real strain of the specimen moreover, as already described by the previous work [1], in the carbon/system the opposite behaviour takes place. However, with the increase of the strain, the FBG sensor $\left(\varepsilon_{\mathrm{FBG}}\right)$ approaches a value closer to the real one in both material and sensor systems.

The difference between the strain given by the Braggmeter and the real strain in the specimen occurs due the different stiffness of materials [9], as well as incomplete adhesion of the FBG sensor to the host material. In the glass/epoxy prepreg system, the stiffness of the FBG sensor is higher when compared to the host material, this will locally increase the stiffness of the specimen, leading to a measurement of a lower strain than the real strain in the sample. The opposite occurs, if the stiffness of the FBG sensor is lower than the host material, such as in the carbon/epoxy prepreg system. Also, is possible to observe a bigger mismatch of the strain value measured to the real strain value when the difference of the stiffness between the FBG sensor and the host material is higher, as in the carbon fiber/ epoxy set.

In order to develop a method to determinate the real strain in the composite laminate from the effective strain sensed by the FBG sensor, a correction factor (Calibration rule) was already established for the carbon fiber/epoxy set in previous work [1], the same rule for the glass fiber/epoxy set was established here and a comparison between them carried out.

This correction factor was defined by the best fit of the data of the ratio $R_{\text {sample/FBG }}$ for the five specimens tested with a 2nd order polynomial. In Table 2 and Fig. 3, the coefficients and the plots of the 2nd order polynomial fittings for all the five tested specimens are, respectively, presented.

In our case (glass fiber/epoxy), the 2nd order polynomial of the specimen number 2 was chosen, because it was the one presenting a better agreement for all the relevant domain, thus suggesting better results. It should be noted that this correction factor is only valid for this combination of FBG sensor and host material.

The experimental procedures to determinate the correction factor and to acquire the correct strain were already described in the previous work [1], although the procedure for the glass fiber/epoxy prepreg can be summarized by the following steps:

Table 2

Parameters of the Polynomial Fit of the Ratio Rsample/FBG presented in Fig. 2, for glass/epoxy prepreg and carbon/epoxy prepreg system.

\begin{tabular}{|c|c|c|c|c|c|c|c|c|}
\hline & \multicolumn{8}{|c|}{ Polynomial fit } \\
\hline & \multicolumn{8}{|c|}{$\mathrm{R}_{\text {sample } / \mathrm{FBG}}=\mathrm{B}_{1}+\mathrm{B}_{2} \times \varepsilon_{\mathrm{FBG}}+\mathrm{B}_{3} \times\left(\varepsilon_{\mathrm{FBG}}\right)^{2}$} \\
\hline & \multicolumn{4}{|c|}{ Glass fiber/epoxy } & \multicolumn{4}{|c|}{ Carbon fiber/epoxy } \\
\hline & $\mathrm{B}_{1}$ & $\mathrm{~B}_{2}$ & $\mathrm{~B}_{3}$ & Adj.R-Square & $\mathrm{B}_{1}$ & $\mathrm{~B}_{2}$ & $\mathrm{~B}_{3}$ & Adj.R-Square \\
\hline Specimen 1 & 1.12 & 16.83 & -1111.36 & 0.956 & 0.79 & 19.375 & -945.72 & 0.92 \\
\hline Specimen 2 & 1.19 & -17.89 & 698.87 & 0.974 & 0.81 & 19.792 & -1471.91 & 0.79 \\
\hline Specimen 3 & 1.01 & 35.39 & -2057.12 & 0.951 & 0.78 & 29.18 & -2108.15 & 0.92 \\
\hline Specimen 4 & 1.13 & 35.63 & -3538.69 & 0.756 & 0.81 & 42.50 & -4548.49 & 0.88 \\
\hline Specimen 5 & 1.23 & -38.92 & 3127.06 & 0.715 & 0.88 & 35.60 & -2854.6 & 0.87 \\
\hline
\end{tabular}



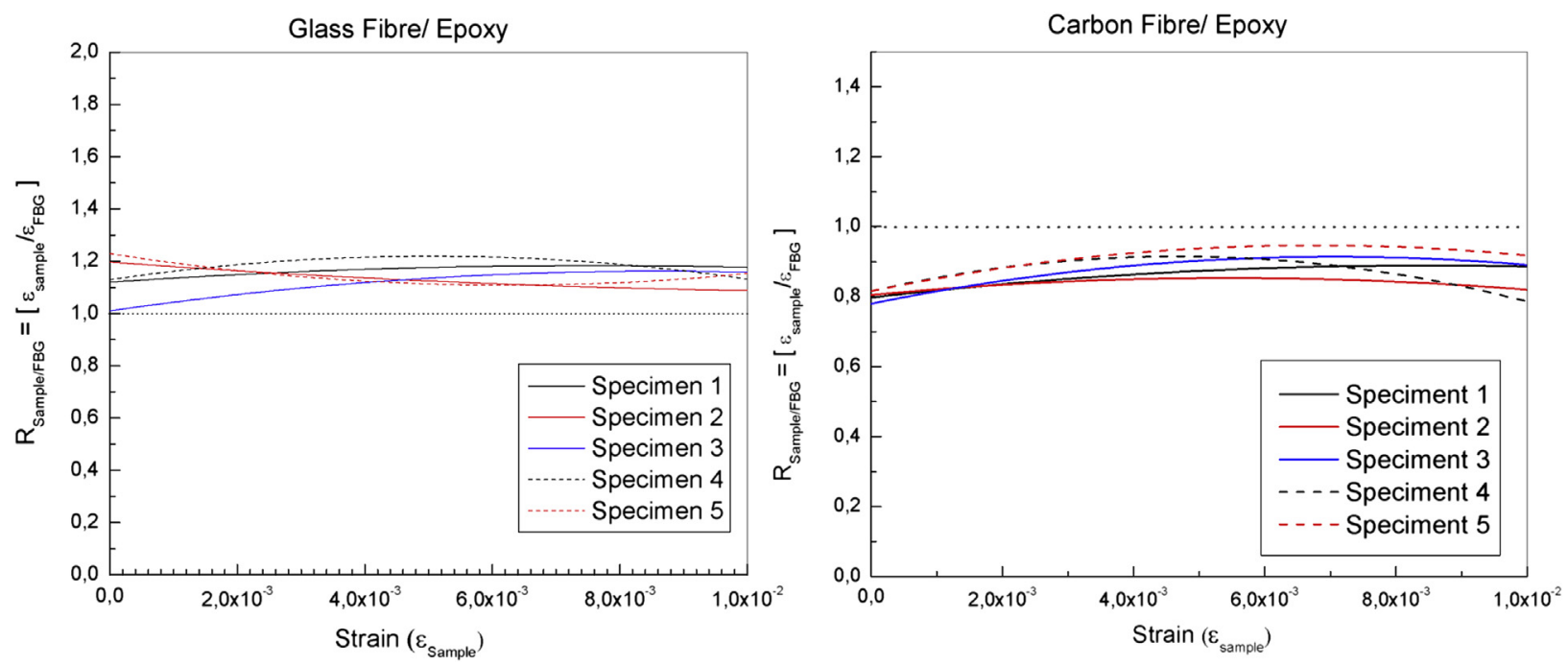

Fig. 3. 2nd order polynomial fits of the ratio data for all tested specimens.

1. Measure the wavelength shift, given by $\Delta \lambda$, using the acquisition system and convert this value to strain (not rectified) $\varepsilon_{\mathrm{FBG}}$, as shown in Equation 3.

$\varepsilon_{F B G}=S_{\Delta \lambda \rightarrow \varepsilon} \times \Delta \lambda=\frac{1}{1.1 \times 10^{3}} \times \Delta \lambda$

2. Calculate the correction factor "FC", given by Equation 4 defined by the 2 nd order polynomial presented in the Table 2 .

$C F=1.19-17.89 \times \varepsilon_{F B G}+698.87 \times\left(\varepsilon_{F B G}\right)^{2}$

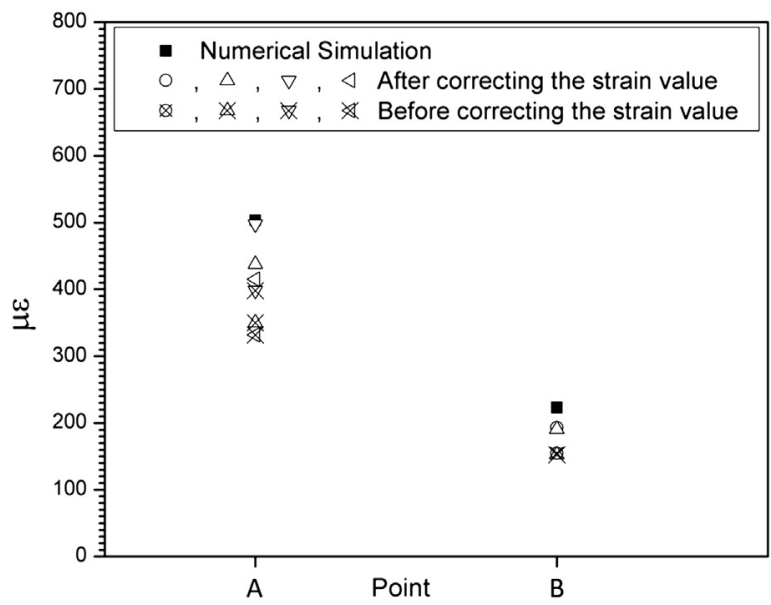

Fig. 4. Comparison of the strain measured by the FBG sensor before and after correcting the strain value.
3. Finally, correct the value of strain measured by the FBG sensor, using Equation 5, obtaining a more accurate value of real strain of the specimen, given by $\varepsilon^{\prime}$.

$\varepsilon^{\prime}=\varepsilon_{F B G} \times \frac{1}{C F}$

\section{4. .Application of the calibration rule in the monitoring of COPV}

As an application of the calibration rule, it was decided to embed the FBG sensor in a pressure vessel produced by filament winding, as shown in Fig. 4. In Fig. 5, the application of the calibration rule to the monitoring of a composite overwrapped vessel (COPV) at an internal pressure of 20 bar is presented and, as shown in Table 3, a significant improvement of the strain measured after the correction factor been applied was verified.

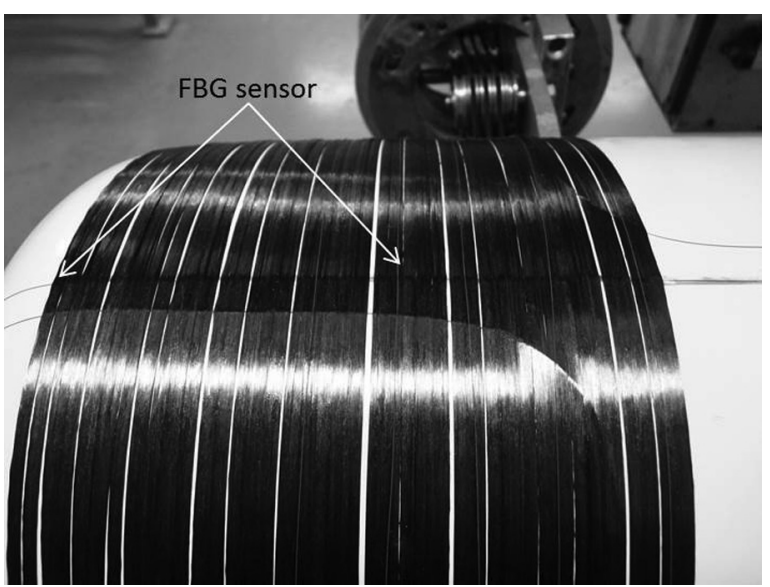

Fig. 5. View of the FBG sensor positioning in the COPV. 
Table 3

Correction factor applied to the monitoring of a COPV.

\begin{tabular}{|c|c|c|c|c|}
\hline \multirow{4}{*}{ Numerical simulation } & \multicolumn{4}{|c|}{$\begin{array}{l}\text { Correction factor applied to the } \\
\text { monitoring of a COPV }\end{array}$} \\
\hline & \multicolumn{2}{|c|}{ Point A $(\mu \varepsilon)$} & \multicolumn{2}{|c|}{ Point B $(\mu \varepsilon)$} \\
\hline & 503.551 & & 223.13 & \\
\hline & Before CF & After CF & Before CF & After CF \\
\hline FBG 1 & 345.85 & 437.787 & 175.41 & 190.66 \\
\hline FBG 2 & 353.55 & 447.54 & 178.39 & 193.34 \\
\hline FBG 3 & 327.94 & 415.18 & & \\
\hline
\end{tabular}

\section{Conclusions}

From the analysis of the results, several conclusions were drawn. The quality and reliability of the values outputted by the FBG sensor strongly depend on the difference between the material properties of the FBG sensor and the host material. This means, that perfect bounding between the FBG sensor and the host material does not occur, leading to a mismatch between the real strain in the specimen and that outputted. Hence, a calibration must be done for each sensor/host pair.

For the host materials applied in this study, correction factors were determined:

$$
\begin{gathered}
\left(C F=1.12+16.83 \times \varepsilon_{F B G}-1111.36 \times\left(\varepsilon_{F B G}\right)^{2} ;\right. \\
\left.C F=1.19-17.89 \times \varepsilon_{F B G}+698.87 \times\left(\varepsilon_{F B G}\right)^{2}\right) .
\end{gathered}
$$

Also, we concluded that a greater difference of the stiffness between the FBG sensor and the host material will lead to a higher mismatch of the strain value measured compared to the real strain, as in the carbon fiber/epoxy set compared with the glass fiber/epoxy set.

The practical implications of these conclusions are that, whenever a new application of FBG sensors to measure strains in composite structure is set, a calibration procedure must be done in order to have reliable measurement throughout the service life of the part or component to be monitored.

\section{Acknowledgements}

The authors acknowledge the Portuguese Foundation for Science and Technology (FCT) for funding the project PTDC/EME-PME/102806/2008 within which this study was conducted.

\section{References}

[1] G. Pereira, C. Frias, H. Faria, O. Frazão, A.T. Marques, On the improvement of strain measurements with FBG sensors embedded in unidirectional composites, Polymer Testing 32 (2013) 99-105.

[2] I. Bennion, J.A.R. Williams, L.Z. Hang, UV-written in Fiber Bragg Gratings, Optical and Quantum Electronics, vol. 28, Springer, 1996. pp. 93-135.

[3] B. Glisic, D. Inaudi, Sensing tape for easy integration of optical fiber sensors, Composite Structures B (2003).

[4] R. de Oliveira, C.A. Ramos, A.T. Marques, Health monitoring of composite structures by embedded FBG and interferometric FabryPérot sensors, Computers \& Structures 86 (2008) 340-346.

[5] J. Degrieck, W. De Waele, P. Verleysen, Monitoring of fiber reinforced composites with embedded optical fiber Bragg sensors, with application to filament wound pressure vessels, NDT \& E International 34 (2001) 289-296.

[6] K.-T. Lau, L. Yuan, L.-M. Zhou, J. Wu, C.-h. Woo, Strain monitoring in FRP laminates and concrete beams using FBG sensors, Composite Structures 51 (2001) 9-20.

[7] K.S.C. Kuang, R. Kenny, M.P. Whelan, W.J. Cantwell, P.R. Chalker, Embedded fiber Bragg grating sensors in advanced composite materials, Composites Science and Technology 61 (2001) 1379-1387.

[8] E. Marin, L. Robert, S. Triollet, Y. Ouerdane, Liquid resin infusion process monitoring with superimposed fiber Bragg grating sensor, Polymer Testing 31 (2012) 1045-1052.

[9] G. Luyckx, E. Voet, W. Waele, J. Degrieck, Multi-axial strain transfer from laminated CFRP composites to embedded Bragg sensor: I. Parametric study, Smart Materials and Structures 19 (2010).

[10] A. Vieira, R. de Oliveira, O. Frazão, J.M. Baptista, A.T. Marques, Effect of the recoating and the length on fiber Bragg grating sensors embedded in polymer composites, Materials \& Design 30 (2009) 1818-1821.

[11] A.W. Morey, G. Melte, W. Glenm, Fibre Optic Gratings Sensors, Fiber Optic Lasers Sensors VII (1989), 1169-98-107. 